\title{
THE OBSERVATION AND ANALYSIS OF DIFFICULTIES OF MOROCCAN FIRST BACCALAUREAT YEAR IN PHYSICAL AND CHEMISTRY SOLVING PROBLEM
}

\author{
[OSERVATION ET ANALYSE DES DIFFICULTES DES ELEVES DE \\ PREMIERE ANNEE BACCALAUREAT MAROCAIN EN \\ RESOLUTION DE PROBLEMES DE PHYSIQUE-CHIMIE]
}

\author{
Ali Ouasri - Moulay Abderrahim El Mhammedi
}

doi: 10.18355/PG.2018.7.1.17

\begin{abstract}
The propose of this study is to observe and analyze the difficulties that pupils (17-18 and) of the Moroccan first baccalaureate year class meet in problems solving activities of physical sciences. The analysis of pupils writing reproductions and the observations of problems solving sessions for these class revealed that pupils in problems solving situations were in difficulties that can be due to the pupils posture face to qualitative data, to the representations of pupils on some concepts and notions and to the incapacity of a number of pupils to traduce the sentences of mathematical content to adequate formula that can serve to solving problems in physic and chemistry
\end{abstract}

Key words

problems solving, teaching, learning, didactic, concept

\section{Résumé}

Cet article se propose d'observer et analyser les difficultés rencontrées par les élèves (17-18 ans) de classes de première année baccalauréat marocain, en activités de résolution de problèmes des sciences physiques. L'analyse des productions écrites et les observations des séances de résolution de problèmes de ces classes montrent que les élèves, en situation de résoudre un problème, faisaient face à des difficultés qui semblent être dues à leur posture face aux données qualitatives, à des représentations de ces élèves de certains notions et conceptions et à l'incapacité, d'un nombre important d'élèves, de traduire des phrases de contenu mathématique à des formules servant à la résolution de problèmes en physique et chimie

\section{Mots clés}

résolution de problèmes, enseignement, apprentissage, didactique, concept

\section{Introduction}

Parmi les activités proposées aux élèves et étudiants dans le cadre d'un enseignement-apprentissage, la résolution de problèmes occupe une place importante (Fagnant et Vlassis, 2010 ; Rober et Marc, 2013). Elle permet non seulement un entraînement à l'utilisation des notions nouvellement acquises, mais joue aussi un rôle important dans l'autorégulation des élèves (Poissant 
et al., 1994). La résolution de problèmes tient une place qui ne se limite plus à l'évaluation des élèves, mais concerne directement les enseignements et les apprentissages (Rey et al, 2012 ; Fagnant et Vlassis, 2010 ; Mazouze et Lounis, 2015 ; Mazouze, 2016, Ouasri, 2016). D’ailleurs, Tardif (1999) a affirmé que la résolution de problèmes devrait constituer la pierre angulaire du curriculum scolaire. Les activités de résolution de problèmes présentent des moments privilégiés permettant, à l'apprenant, de s'exercer à la mobilisation de ses acquis, à la critique, à l'émission des hypothèses, à la conception des notions et à l'interprétation des résultats. Ces activités aident à l'apprentissage de nouveaux concepts et procédures scientifiques et engagent les élèves à se construire des connaissances ayant du sens et qui peuvent être mobilisables face aux nouvelles situations (Fagnant et Vlassis, 2010).

Du point de vue psychologique, il y a problème chaque fois qu'un but ayant été fixé, on n'a pas encore identifié les moyens de l'atteindre (Gagné, 1985) ou encore, il y a problème lorsqu'un obstacle sépare notre état actuel d'un autre désiré (Glover et al., 1990). Ces définitions mettent en évidence deux pôles fondamentaux du problème: la situation de départ et le but à atteindre. Résoudre un problème revient donc à trouver les moyens et leurs combinaisons efficaces pour aller de la situation de départ au but à atteindre. Poissant et al. (1994) avaient défini ce qu'est un problème et les différentes étapes de sa résolution, toute en insistant sur la composante d'autorégulation inhérente au processus de résolution de problèmes. Cette composante cruciale permet une remise en cause constante des actions de l'apprenant, et sa maîtrise permet de rendre celui-ci plus autonome à l'égard de ses apprentissages.

Une des questions qui a d'abord mobilisé les chercheurs était d'identifier des stratégies de résolution applicables dans n'importe quel contexte. Les travaux de Newell et Simon (1972) ont constitué une étape marquante de la recherche sur la résolution de problèmes, dans le cadre de la psychologie cognitive. Ces deux chercheurs avaient développé pendant une quinzaine d'années un programme de recherche tout en jetant les bases de l'intelligence artificielle (IA).

La résolution de problème a fait l'objet de plusieurs recherches en didactique des sciences en général et en didactique de la physique et de la chimie en particulier (Caillot et al., 1990 ; Goffard 1994; Sall 2002; Roditi 2006; Pallascio, 2005; Streveler et al, 2008 ; Fagnant et Vlassis, 2010 ; Mazouze et Lounis, 2015 ; Mazouze, 2016). Certains de ces travaux avaient porté sur la mise en œuvre d'aides didactiques et cognitives servant d'outils à l'apprentissage de la stratégie de résolution de problèmes de physique pour guider le processus d'élaboration de la représentation du problème. A ce propos, Goffard (1994) avait développé la résolution de problèmes de physique suivant trois orientations:

- Les stratégies, les raisonnements et les difficultés des élèves en résolution de problèmes.

- L'étude des différents aspects des «situations problèmes » offertes aux élèves. 
- Les aides à apporter aux élèves pour développer leurs stratégies en matière de résolution de problèmes d'une part et les faire apprendre en résolvant des problèmes d'autre part.

Par ailleurs, Sall (2002) a fait ressortir les traits dominants dans les conceptions des professeurs en résolution de problèmes et la place importante que méritent d'occuper aussi bien de tels concepts transversaux que ceux de types disciplinaires. Les travaux de Sall se sont distingués de plusieurs travaux antérieurs qui avaient porté sur le "pôle élèves" par ce qu'il considérait comme cible le "pôle enseignant".

Cet article se propose d'étudier les difficultés de l'apprentissage par la résolution de problèmes des élèves aux lycées marocains; il porte ainsi sur cinq parties. La première partie aborde la problématique de la résolution des problèmes, en particulier dans le contexte scolaire marocain. La deuxième partie développe le cadre conceptuel de la résolution des problèmes dans une approche cognitiviste, en lien au constructivisme en didactique. La troisième partie illustre la méthodologie de recherche adoptée, basée essentiellement sur les observations de classe et l'analyse des productions des élèves soumis aux activités de résolutions de problèmes de physique et de chimie. Dans la quatrième partie, sont présentés les résultats de recherche qui seront, enfin, discutés dans la cinquième partie avant de conclure notre propos.

\section{Contexte et problématique}

Dans le contexte marocain, l'outil d'évaluation le plus utilisé par les enseignants est la résolution de problèmes par les élèves. Les performances des élèves marocains des sections scientifiques ne sont pas satisfaisantes, surtout aux épreuves de physique au Baccalauréat. L'échec d'un nombre important d'élèves devant des problèmes de physique est une chose évidente (Ouasri, 2016, 2017a, 2017b, 2017c). Cet échec semble être attribué à l'élève lui-même, à l'enseignant, ou aux deux ensembles. D'où notre intérêt à l'étude de certaines origines des difficultés des élèves marocains en vue d'améliorer leur performance dans la résolution de problèmes en physique et en chimie.

Le cadre problématique de cette étude place les élèves au centre de nos préoccupations en adéquation avec la psychologie de l'apprentissage, y compris le constructivisme qui permet d'éclairer les questions liés à l'apprentissage et à la résolution de problèmes.

Vue l'importance capitale du rôle de l'enseignant dans le guidage ou l'aide apportée aux élèves en résolution des problèmes, son intervention ne devrait donc pas passer sous silence. Ainsi, nous nous sommes intéressés à l'observation des professeurs en classes en intéractions avec les élèves en résolution de problèmes. Roditi (2006) s'est intéressé aux pratiques enseignantes en analysant les projets pédagogiques de divers enseignants de mathématiques au niveau de la préparation et l'animation en situation de classe. Certaines études se sont intéressées à comprendre les facteurs qui influencent les pratiques des enseignants (Van der Sandt, 2007) alors que d'autres se sont penchées sur les facteurs qui peuvent expliquer les démarches des élèves (Verschafell et al., 2000). En effet, résoudre un problème demeure toujours une préoccupation majeure aussi bien pour les 
élèves que pour les enseignants qui sont toujours priés de proposer à leurs élèves des problèmes à résoudre.

Quelque soit le type d'apprentissage visé par la résolution de problèmes, il convient de souligner que les objectifs sont intrinsèquement liés au développement de compétences transversales qui contribuent à l'attribution du sens aux contenus, procédures et stratégies de résolution. L'intérêt de proposer aux élèves des problèmes plus au moins complexes est alors de développer ces compétences.

Dans la présente étude, on s'intéresse à éclairer quelques origines des difficultés qui s'opposent à l'apprentissage des stratégies de résolution de problèmes par les élèves de la première année Baccalauréat (trois classes en sciences éxpérimentales et une en sciences mathématiques). Cette étude s'inscrit dans le cadre du courant de recherches centrées sur le paradigme expert/novice ayant comme objectif de faire face à des faiblesses des novices et les aider à développer certaines compétences en résolution de problèmes. Elle porte aussi sur la l'analyse des différentes étapes (tâches) accomplies par les élèves lors de la résolution de problèmes en dénombrant le nombre de réponses réussies, échouées et non traités sans pour autant évoquer le processus d'élaboration de ces réponses, i.e. sans toucher au contenu informatif des propos évoqués dans le processus. Voila donc la problématique autour de laquelle s'article la présente étude.

Mais auparavant, qu'entends-t-on par la résolution de problèmes? Quelles en sont les principales étapes? Quels sont les courants de recherches qui s'intéressaient à la résolution de problèmes? Et quelles sont les spécificités de la physique et de la chimie en lien avec la résolution de problèmes?

\section{Cadre conceptuel}

La didactique des sciences, en particulier celle de résolution de problèmes, convoque plutôt le constructivisme qui met l'apprenant au cœur $\mathrm{du}$ processus de construction du savoir procédural ou déclaratif. Les contributions de Piaget et de Vygotski, ont apporté des éclairages décisifs sur le développement cognitif, malgré leurs itinéraires croisés. Malgré le succès réservé aux conceptions piagétiennes du développement de la pensé, les tentatives de réinvestissement dans un champ disciplinaire en ont révélé des limites certaines. Le paradigme piagétien n'a pas eu d'influence directe sur la résolution de problèmes dans une perspective didactique en termes des difficultés d'appropriation des savoirs et des démarches intellectuelles mis en jeu dans un champ disciplinaire spécifique tel que la physique et la chimie.

\subsection{La didactique de la résolution de problème}

La résolution de problèmes devient de plus en plus un outil d'enseignement et d'apprentissage à usage multiples. Au niveau didactique, la résolution de problème est prise en charge à travers trois perspectives: l'évaluation, l'apprentissage et la recherche (Sall, 2002). Au scolaire, nous nous intéressons seulement aux deux premières perspectives:

- Outil d'évaluation: la résolution de problème est ici un outil principal dans une approche globale pour rectifier des lacunes, apporter des corrections aux représentations après enseignement et approfondir l'esprit d'analyse et de 
raisonnement. Dans l'enseignement de la physique-chimie, la résolution de problème est considérée comme l'activité intellectuelle à travers laquelle on évalue les compétences des élèves. Il y a compétence lorsqu'un élève est capable de mobiliser des ressources à l'occasion d'une tâche originale. Mais on ne peut nommer compétence la simple possession de ressources qu'un élève soit capable, quand on lui en fait la demande, d'exécuter une opération à laquelle on l'a entraîné ou de répéter un énoncé qu'il a mémorisé. Pour qu'il y ait alors compétence, il faut qu'il soit capable de choisir lui-même, parmi les ressources qu'il possède, celle qui convient à la situation présente.

- Activité d'apprentissage des concepts scientifiques: dans l'enseignement/apprentissage de la physique-chimie, les activités de résolution de problème sont organisées soit à l'intérieur du cours sous forme d'exercices d'application, soit dans des séances particulières. A l'intérieur du cours, le professeur après avoir établi une relation de manière théorique ou expérimentale, propose une situation qui permet de mettre en scène les nouveaux concepts par une application d'une nouvelle relation; ce qui favorise mieux l'apprentissage d'une telle relation. L'apprentissage par résolution de problèmes est une approche constructiviste, et l'apprenant y étant véritablement au centre de la construction du savoir (Mazouze \& Lounis, 2015). L'apprentissage par situation-problème est une approche qui se développe de plus en plus compte tenu des modifications de l'environnement éducatif, notamment avec le développement des nouvelles technologies de l'information.

\subsection{Etapes de résolution de problème}

La résolution de problèmes est un processus qui se déroule en différentes étapes qui se succédent dans un ordre plus ou moins variable (Newell et Simon, 1972; Gagné, 1985; Glover et al., 1990; Goffard, 1994; Sall 2002). En général, une convergence apparaît sur l'existence de plus ou moins quatre étapes importantes:

- L'analyse de la situation: met l'accent sur la représentation du problème et sa signification situationnelle. La représentation est le produit de la compréhension du problème. La compréhension renvoie à deux perspectives liées: l'une, épistémique, correspond à l'intégration de la nouvelle information dans les structures cognitives du sujet, l'autre, dite pragmatique, a pour finalité l'identification du résultat à atteindre. La compréhension prise au sens pragmatique renvoie à la notion de tâche qui est au cœur de la résolution de problèmes. Il est important de mettre garde contre toute confusion entre tâche et problème: une même tâche peut être une simple exécution de procédures déjà maitrisées et donc ne pose pas de problème au sujet, alors qu'elle peut constituer un véritable problème pour un autre.

- La résolution: prise en charge par l'espace-problème de Newell et Simon (1972), cette étape qui est un processus, une construction, une dynamique, a pour but de construire un chemin entre la situation de départ et le but à atteindre. En tant que telle, la résolution nécessite non seulement la mobilisation de connaissances générales et spécifiques, mais aussi des opérateurs ou une combinaison d'opérateurs. Les processus suivis face à un 
même problème peuvent donc varier d'un sujet à un autre tout en étant efficaces.

- La validation: consiste à s'assurer que la solution proposée permet d'aboutir au but attendu, mais avec une dimension économique car une solution peut être valide sans être économique. Par exemple, si on demande aux élèves de déterminer expérimentalement la concentration d'une solution (dosage), il est préférable de travailler avec un volume très petit de cette solution au lieu d'en utiliser un grand volume, puisque cela donne le même résultat.

- La communication des résultats: cette dernière étape qui est particulièrement importante dans le contexte scolaire permet de contrôler une dernière fois la valeur de la solution.

$\mathrm{Au}$ cours de ces étapes, les experts recourent davantage à des stratégies de résolution que les novices.

\subsection{Courants de recherches en résolution de problèmes}

Les travaux effectués sur la résolution de problèmes correspondent aux plusieurs courants de recherches. Nous retenons ici trois courants de recherches en résolution de problèmes en lien avec la problématique de la présente étude.

- Recherches centrées sur le paradigme expert/novice (Chi et al., 1981; Larkin et Rief, 1979) dont l'approche de résolution est basée sur la catégorisation des sujets en face d'un problème. Cette catégorisation s'appuie sur l'observation de différences significatives du comportement entre les sujets qui réussissent le plus à résoudre les problèmes et ceux qui n'y réussissent pas. Sur le plan pédagogique, la le passage du novice à l'expert, ou le développement des compétences en résolution de problèmes, semble être cruciale pour faire face à la faiblesse des élèves en résolution de problème.

- Recherches destinées à développer les compétences des élèves (Noirfalise et Porte, 1990; Verschaffel et al, 2000; Rey et al, 2012) dont l'objectif est l'apprentissage du processus de la résolution de problèmes envisagés comme un processus complexe de modélisation; i.e. l'apprentissage de stratégies heuristiques (souvent informelles) et métacognitives inhérentes aux différentes phases de la résolution. La résolution de problèmes contribue à la formation des élèves aux compétences de la démarche scientifique qui ne peut être que progressive et construite avec le temps. Il s'agit alors d'analyser le processus de la résolution en mettant l'accent sur l'importance des phases qui structurent la démarche de l'expert. Les activités cognitives de l'élève sont également prises en compte dans l'expérimentation. Le premier pas pour l'élève confronté à une résolution de problèmes est de décrypter et s'approprier le problème. Après lecture, l'élève doit être en mesure d'expliciter les objectifs du problème proposé. La maîtrise de la compétence "s'approprier" (Noirfalise et Porte, 1990) demande à l'élève de questionner les documents pour identifier quelles informations ils apportent (en regard de la problématique), pour retranscrire les données utiles et commencer à les articuler entre elles et avec ses propres connaissances. En deuxième lieu, il s'agira d'analyser ces informations en les complétant par les lois et les principes qui semblent nécessaires pour modéliser la situation (Noirfalise et 
Porte, 1990). Cette phase préalable d'appropriation et d'analyse est indispensable à la construction de la stratégie; c'est l'étape dite de décontextualisation d'un problème où le codage de l'information s'appuie sur un formalisme dédié (lexical, schématique, mathématique..). Cette étape, cruciale pour faire comprendre aux élèves la manière dont la science répond aux questions posées, constitue une des difficultés majeures dans l'enseignement de la physique-chimie.

- Recherches axées sur la résolution de problème comme contexte d'apprentissage n'ont pas pour objectif exclusif d'apprendre aux élèves à résoudre des problèmes, mais l'apprentissage des concepts, des modèles et de la modélisation. Le point de départ est la construction du savoir de l'élève à partir de leurs conceptions, avec la prise en compte de la représentation. Les recherches de Gil Perez et Martinez-Terregosa (1983) ont porté sur des problèmes ouverts, sans données. Aussi, Weil-Barais et Lemeignan (1990) avaient effectué des recherches sur l'apprentissage des modèles et concepts en mécanique en modélisant des situations expérimentales. L'étude de ces concepts implique des analyses épistémologiques et psychologiques. La suite de la résolution de problèmes est alors destinée à la construction et l'apprentissage de nouveaux concepts (Fabre, 1997; De Vecchi et CarmonaMagnaldi, 2002; Pallascio, 2005; Fagnant et Vlassis, 2010).

L'examen de la littérature révèle une évolution progressive des recherches axées sur le paradigme "expert/novice", vers des recherches visant à faire de la résolution de problèmes des activités d'apprentissage, de modélisation et de construction des concepts scientifiques. Dans cette étude, nous nous sommes opté à ces tendances, notamment au paradigme expert /novice.

Nous discutons dans ce qui suit les spécificités de la physique et de la chimie, sur la didactique de la résolution de problème et les différentes perspectives de cette didactique.

\subsection{Les spécificités de la physique et de la chimie}

La physique et la chimie sont des sciences de modélisation et de mesurage. La modélisation permet de se distancer par rapport à un monde réel par essence complexe et de générer des connaissances scientifiques. Celles-ci sont toujours approchées, partielles et provisoires. Pour être pertinent, l'enseignement doit impliquer l'apprenant dans le processus de construction des sciences. Et pour accompagner cette construction progressive des connaissances par l'apprenant, l'enseignant devrait assurer une fonction de médiateur.

Il est à noter que toute situation d'enseignement met en présence l'enseignant, l'élève et le savoir à enseigner qui constituent le pôle sociologique, le pôle psychologique et le pôle épistémologique, respectivement. La didactique prend en charge à la fois ces trois pôles et leurs intéractions. La didactique de la physique et de la chimie s'intéresse plus particulièrement à la relation mise en jeu entre l'élève et le savoir. L'enseignant de physique-chimie est également en intéraction avec le savoir et il est constamment confronté, en sa qualité de médiateur, au processus de construction. D'où certaines spécificités qui caractérisent la physique et la chimie. 
La résolution de problème peut être abordée sous l'angle psychologique en relation avec le processus d'acquisition ou de construction du savoir scientifique. C'est pourquoi la résolution de problèmes est très valorisée dans les activités scolaires destinées à guider l'apprentissage ou à évaluer les acquisitions des élèves, surtout pour la physique et la chimie. La résolution de problème dans l'enseignement-apprentissage de la physique et la chimie convoque au moins deux champs théoriques qui interfèrent dans l'analyse des processus éducatifs: la psychologie et l'épistémologie des sciences (Sall, 2002); la résolution de problème peut être décrite comme une activité cognitive finalisée au service d'une tâche à effectuer dans une situation donnée (Richard, 1990). Elle fait appel à une activité psychique et à des processus intellectuels mettant en jeu des connaissances (Tardif, 1999). Celles-ci sont acquises ou construites par le biais des processus intellectuels antérieurs.

Tout ce qui nous intéresse dans cet article, c'est l'étude des tâches (réussies, échouées ou non traités) sans pour autant aller développer l'analyse les activités psychiques et les processus intellectuels mis en jeu par les élèves dans la réalisation ou non d'une telle tâche.

\section{Méthodologie}

La méthodologie adoptée dans cette étude est axée sur les observations des séquences d'activités en classes; elle met implicitement l'accent sur les trois niveaux des interactions en classes, illustration d'une socio-construction du savoir. Cette méthodologie tient compte non seulement des actions réalisées par les élèves en interaction avec l'objet de savoir dans leurs activités de résolution de problèmes, mais aussi, quoiqu'observés superficiellement, des actes posés par les enseignants pour les y aider ainsi que les interactions entre élèves eux-mêmes.

Ainsi, nous nous sommes appuyés d'une part sur l'observation des comportements des apprenants lors des situations de résolution de problèmes en intéractions avec des professeurs qui cadrent leurs actions et d'autre part sur l'analyse des reproductions des élèves sur des problèmes de physique et de chimie qui leur ont été soumis. Les analyses proposées pour les reproductions des élèves portent essentiellement sur le dénombrement des nombres des réponses obtenus en termes de réussite, échec et non traitée. Dans ce qui suit, nous allons définir la population cible, les méthodes d'investigation et les instruments de collecte de données.

\subsection{Population cible}

Cette étude réalisée au terme de l'année scolaire 2012-2013 avait pour cible environ 100 élèves (18-20 ans) appartenant aux quatre classes (A, B, C, D) de première année Baccalauréat du lycée Lala Aicha situé à la Rue Moulay Ali Chérif de Rabat (Maroc). Chacune de ces classes totalise quatre heures de cours hebdomadaires (deux séances de deux heures) réparties du lundi au vendredi. Les élèves travaillent en journée continue de 8 heures à 17 heures avec une pause de deux heures, de 12 heures à 14 heures.

Outre la collecte des reproductions écrites des élèves, nous avons soumis les élèves de ces classes et leurs professeurs à des observations lors des séances

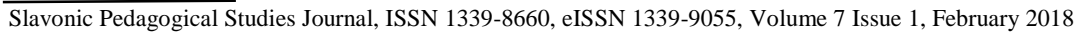


de résolution de problèmes organisés aux profits des élèves. Dans cette étude nous retenons à titre d'exemple les résultats relatifs à deux classes, soit à deux professeurs.

Tous les professeurs ont comme diplôme une licence (diplôme sanctionnant trois ans des études universitaires), en physique ou en chimie, qui leur avait permis sur sélection et concours (écrit et oral) d'accéder à une formation professionnelle d'une année avant d'aller exercer leurs fonctions de professeurs secondaires qualifiants aux lycées marocains.

\subsection{Méthodes d'investigation et instruments}

Pour pouvoir analyser les difficultés des élèves en activités de résolution de problèmes, nous avons procédé à:

- L'observation des séances de résolution de problèmes dirigées par les professeurs en vue de voir les acteurs directement en œuvre et de faire ainsi une analyse des difficultés détectées chez les élèves. La collecte des données s'est faite par prise de notes d'observation pour chaque professeur dans sa classe en utilisant une grille d'observation d'une séance de résolution de problèmes 1 et 2 illustré dans le tableau 1 (Annexe 1). Le recours à l'observation dans la pratique de la classe permet de décrire les activités visibles manifestées par l'enseignant et les élèves en situation de résolution de problèmes; ce qui nécessite un instrument de recueil et d'interprétation des renseignements relatifs à l'animation pédagogique qui a lieu en classe.

Cette grille s'inspire du modèle de grilles proposées par D'Hainaut (1988) pour l'observation du comportement pédagogique des élèves et d'enseignant dans une classe. On note que la mobilisation et la résolution de problèmes sont les deux niveaux supérieurs des opérations cognitives proposées par D'Hainaut. L'observation des professeurs en intéractions avec les élèves lors des séances de résolution de problèmes nous permet de remplir la grille d'observation pour plusieurs professeurs.

- L'analyse des productions écrites des élèves (tâches) en situation de résolution de problèmes afin de déceler certaines des difficultés auxquelles sont confrontés les élèves quand ils sont en train de résoudre de problèmes de physique et de chimie. L'analyse de tâches réalisées par les élèves a été fait à la loupe de deux grilles d'analyse de contenus illustrés dans les tableaux 2 et 3 (Annexe 2) que nous avons construites et adoptées conformément aux questions posées dans les problèmes 2 et 3 soumis aux élèves. Ces grilles servent d'instruments pour examiner, diagnostiquer les blocages et les erreurs rencontrées par les élèves lors de la résolution de problèmes afin de proposer certaines remédiations.

\subsection{Modalités d'investigation}

Les problèmes utilisés comme outils d'investigation dans la résolution de problème ont été choisis dans des manuels scolaires où les professeurs ont l'habitude de puiser pour la confection de leurs séries d'exercices. Ils ne sont ni trop faciles ni trop difficiles.

\subsubsection{Observation des séances de TD}


Le problème 1 de physique non utilisé pour l'analyse de tâches est soumis aux élèves des classe $\mathrm{A}, \mathrm{B}, \mathrm{C}$ et $\mathrm{D}$ pour l'observation des élèves en interaction avec les professeurs lors des séances de résolution de problèmes.

Le problème 2 de chimie, déjà donné aux élèves des classes $\mathrm{A}$ et $\mathrm{B}$ pour l'analyse de tâches est soumis à nouveau aux élèves des classe $\mathrm{C}$ et $\mathrm{D}$ pour l'observation des élèves en interaction avec les professeurs lors des séances de résolution de problèmes.

Chaque classe avait été prise en charge par un professeur. Pour renforcer l'apprentissage des élèves et les préparer aux séances d'observation, les professeurs avaient consacré aux classes des séances de soutien, de deux heures, par semaine afin de travailler avec leurs élèves des rappels de cours et des corrections des séries d'exercices et de problèmes.

Pour chaque classe, nous avons effectué des observations des séances de résolution de problèmes menées par le professeur concerné en présence des professeurs d'autres classes comme des observateurs chargés d'apporter leurs notes d'observation que nous tenions aussi en compte dans le remplissage de la grille d'observation (tableau 1).

A la fin de chaque séance, on note des commentaires, des appréciations et des suggestions en vue d'en profiter pour déceler certaines faiblesses des professeurs; ce qui nous permet d'apporter certaines régulations à leurs comportements en vue de surmonter ces faiblesses. Il est à noter qu'aucune problématique ne se posait, aucun fait notoire, aucun incident n'est venu perturber le déroulement normal des séances.

Au niveau de la discussion relative à l'observation des séances de résolution de problèmes 1 et 2 , nous ne retiendrons que deux cas :

- Problème 1 de physique (champ magnétique) pour la classe A.

- Problème 2 de chimie (lecture d'une formule chimique) pour la classe B.

\subsubsection{Analyse de tâches}

Nous avons collecté les productions écrites des élèves de chaque classe lors des séances de résolution de problèmes 2 et 3 . Le problème 2 de chimie portant sur "la lecture d'une formule en chimie organique" est soumis aux classes $\mathrm{A}$ et $\mathrm{B}$ (50 élèves). Le problème 3 de physique concernant respectivement le "comportement global d'un circuit électrique" et le "transfert d'énergie dans un circuit électrique" est soumis aux classes C et D (48 élèves). L'analyse des tâches est développée sur la base du nombre des réponses obtenues (réussites, échecs et non traités).

\section{Résultats}

Pour l'analyse des tâches, les élèves de toutes les classes expérimentées ont fini leurs tâches avant la fin du temps imparti, soit une heure. Dés le début, on expliquait aux élèves qu'ils ne pouvaient pas sortir avant l'heure pour éviter que, par envie de sortir très vite, certains élèves en arrivent à arrêter leur travail et donner envie aux autres d'en faire de même.

\subsection{Observation des élèves en séances de résolution de problèmes} L'observation des élèves et leurs professeurs en classes lors des séances de résolution de problèmes est faite sur la base de la grille donnée dans le 
tableau 1 (Annexe 1). Nous présentons ci-après à titre d'exemple les comportements observés pour deux classes A et B.

La classe A: on choisit ici de suivre le comportement des élèves qui sont mis face au problème 1. Au début, le professeur a accordé aux élèves dix min pour réfléchir; ensuite un élève est passé au tableau pour pouvoir répondre à la première question. Les choses vont bien, et c'est à la troisième question que l'élève, au tableau, se confronta aux difficultés puisque la réponse à cette question nécessite la mobilisation, chez l'élève, de ses acquis en termes de connaissances et compétences afin qu'il puisse élaborer un formalisme mathématique à partir d'une phrase de contenu mathématique. A ce moment où tous les autres élèves restaient bloqués, le professeur leur avait présenté certaines explications afin de les aider à traduire le contenu mathématique de la dite phrase en une formule leur permettant de se mettre à nouveau dans le processus d'apporter une solution à ce problème. Parvenus à l'écriture de l'équation " équation de première degré", les élèves ont besoin de retirer la valeur de $\mathrm{x}$ de l'équation. L'élève au tableau n'arrive pas à apporter une solution à ce problème. Le professeur a alors fait participer d'autres élèves de la classe, qui malgré leur enthousiasme et leur participation chaleureuse, la majorité d'entre eux n'ont pas pu non plus apporter de solution au problème posé. Seuls deux parmi eux (soit 2 sur 24 élèves) ont été capables de donner une réponse correcte. On constate alors que les élèves ont un manque énorme au niveau du formalisme mathématique. Ils ont donc besoin d'un soutien consistant en mathématiques pour qu'ils puissent surmonter leur handicap mathématique et accomplir enfin leur tâche de résolution de problèmes en physique et en chimie.

La classe B: on choisit ici de suivre le comportement des élèves qui sont mis face au problème 2. Signalons ici que le problème avait été préalablement proposé aux élèves avant la séance pour un travail de réflexion à la maison. Au début de la séance, le professeur au tableau commence à lire le problème en leur demandant de souligner les mots et les données clés du problème. Puis, un élève est passé au tableau pour résoudre la première question. En même temps, le professeur vérifie si les élèves avaient résolu leur problème. Dans le cadre de la première question de ce problème (1.1), certains élèves ont indiqué au professeur qu'il y a un autre groupe organique, de même formule brute que celle du composé étudié; il s'agit là d'un cycloalcane saturé. Ensuite, le professeur a posé cette question aux autres élèves pour les mobiliser à participer aux activités de résolution. Les élèves n'ont pas réagit, aucune réponse n'a été apporté par les élèves sauf celle de l'élève qui est au tableau. Le professeur demande aux élèves de revenir à l'énoncé du problème, puis il leur pose la question "que signifie le mot non saturé qui est déjà souligné". Un élève commence à répondre en disant que ce composé n'appartient pas au groupe des hydrocarbures saturés qui sont constitués des alcanes et des cycloalcanes.

\subsection{Analyse des tâches}

Les résultats de l'analyse des tâches (sous forme d'épreuves écrites) exécutées par les élèves des classes $\mathrm{A}$ et $\mathrm{B}$ pour le problème 2 , et les élèves des classes $\mathrm{C}$ et $\mathrm{D}$ pour le problème 3 sont consignés dans les tableaux 2 et 3 . 
Nous avons décomposé les productions des élèves en unités simples, chacune d'elles représente une tâche élémentaire à effectuer dans le processus de résolution du problème (paradigme expert-novice). Dans le problème 2 tout come le problème 3 , nous avons identifié 10 tâches à réaliser. Les résultats obtenus dans les tableaux 2 et 3 ont été traduis en figures 1 et 2 qui illustrent le pourcentage des élèves ayant réussit ou non les tâches.

Tableau 2. Résultats relatifs au problème 2 soumis aux élèves des classes A et B.

\begin{tabular}{|l|c|c|c|}
\hline \multicolumn{1}{|c|}{ Tâches prescrites } & Réussies & Echoués & Non traités \\
\hline $\begin{array}{l}\text { Préciser les groupes organiques } \\
\text { auxquels appartiennent-ils les } \\
\text { composés de formules brutes } \\
\text { suivantes composés }\end{array}$ & 42 & 8 & 0 \\
\hline $\begin{array}{l}\text { Définir les } \\
\text { hydrocarbures saturés et insaturés }\end{array}$ & 38 & 10 & 2 \\
\hline $\begin{array}{l}\text { Déterminer les groupements qui } \\
\text { appartiennent à ce composé } \\
\text { insaturé. }\end{array}$ & 38 & 12 & 0 \\
\hline $\begin{array}{l}\text { Déterminer la différence entre les } \\
\text { chaînes carbonées de ce composé }\end{array}$ & 26 & 22 & 2 \\
\hline Définir l'isomérie de constitution. & 32 & 0 & 18 \\
\hline $\begin{array}{l}\text { Déterminer les isomères de } \\
\text { constitution possibles pour ce } \\
\text { composé }\end{array}$ & 30 & 14 & 6 \\
\hline $\begin{array}{l}\text { Donner les formules semi- } \\
\text { développées. }\end{array}$ & 40 & 8 & 2 \\
\hline Donner l'écriture topologique. & 34 & 10 & 6 \\
\hline Définir les stéréo-isoméries. & 26 & 0 & 24 \\
\hline Déterminer les stéréo-isomères. & 32 & 12 & 6 \\
\hline
\end{tabular}




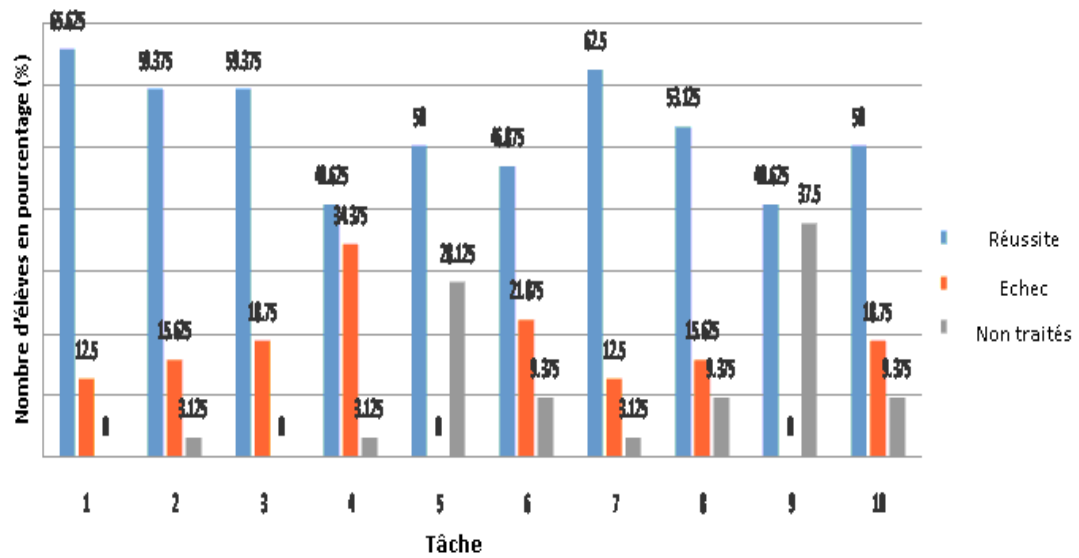

Figure 1. Résultats relatifs au problème 2 traduis en pourcentage d'élèves.

Tableau 3. Résultats relatifs au problème 3 soumis aux élèves des classes C et $\mathrm{D}$.

\begin{tabular}{|c|c|c|c|}
\hline Tâches prescrites & Réussies & Echoués & Non traités \\
\hline Enoncer la loi de maille & 12 & 0 & 36 \\
\hline $\begin{array}{l}\text { En se référant a la loi de maille } \\
\text { écrire une relation entre les } \\
\text { tensions }\end{array}$ & 36 & 4 & 8 \\
\hline $\begin{array}{l}\text { Représenter, sur le schéma, les } \\
\text { tensions } \mathbf{U}_{\mathbf{B C}}, \mathbf{U}_{\mathbf{B D}}, \mathbf{U}_{\mathbf{B A}}\end{array}$ & 26 & 10 & 12 \\
\hline $\begin{array}{l}\text { Calculer la tension à la borne de } \\
\text { dipôle (BD) }\end{array}$ & 24 & 14 & 10 \\
\hline $\begin{array}{l}\text { Indiquer la convention } \\
\text { "récepteur" et "générateur" }\end{array}$ & 8 & 0 & 40 \\
\hline $\begin{array}{l}\text { Déterminer sur le circuit les } \\
\text { générateurs et les récepteurs }\end{array}$ & 20 & 16 & 12 \\
\hline $\begin{array}{l}\text { Calculer la puissance électrique } \\
\text { consommée par les récepteurs }\end{array}$ & 22 & 12 & 14 \\
\hline $\begin{array}{l}\text { Calculer directement la puissance } \\
\text { délivrée par le générateur }\end{array}$ & 16 & 12 & 20 \\
\hline $\begin{array}{l}\text { Calculer, à partir du principe de } \\
\text { conservation de puissance, la } \\
\text { puissance délivrée par le } \\
\text { générateur }\end{array}$ & 10 & 8 & 30 \\
\hline Faire l'application numérique & 14 & 14 & 20 \\
\hline
\end{tabular}




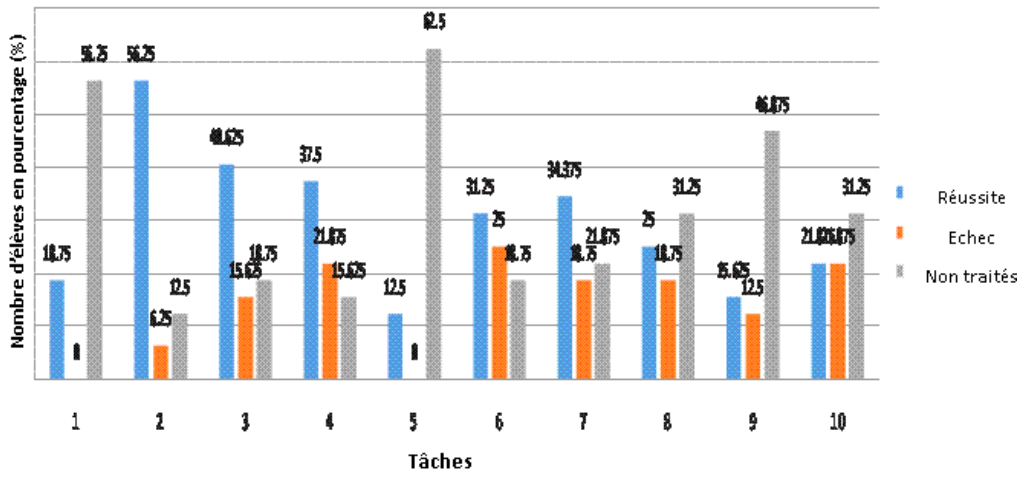

Figure 2. Résultats relatifs au problème 3 traduis en pourcentage d'élèves

\section{Discussions}

5.1. Observation des séances de résolution de problèmes

L'analyse est centrée sur les élèves conformément à l'approche constructiviste qui place l'élève au centre des activités d'enseignement/apprentissage. Pour ce qui des niveaux d'observation (tableau 1), la plupart des professeurs n'ont fait pas apparaitre clairement les objectifs des activités soumises aux élèves, alors qu'ils essaient de susciter l'intérêt des élèves aux activités proposées et les encourager à la participation aux activités de résolution de problèmes. En effet, les professeurs ne communiquent pas seulement avec l'élève au tableau, mais aussi avec tous les autres élèves qui plus ou moins suivent celui qui est au tableau. Lors des activités, les élèves travaillent en groupe pour co-construire des réponses aux questions du problème. De plus, ils se posent des questions entre eux, et ne posent des questions à l'élève au tableau que très rarement. Malgré l'importance de la socio-construction, le déroulement de la séance n'a pas été bien organisé puisque les discussions en groupes se faisaient en parallèle avec le travail effectué soit par le professeur ou par l'élève prenant l'initiative de répondre à une question au tableau.

Dans l'enseignement de la physique/chimie, telle que nous l'avons vu dans le cadre conceptuel, la résolution de problème est considérée comme l'activité intellectuelle à travers laquelle on évalue les compétences des élèves; de ce fait, elle sert d'un outil pour rectifier certaines lacunes observées et apporter des corrections nécessaires aux représentations des élèves après l'enseignement. Les activités de résolution de problèmes observées dans cette étude n'ont pas eues cette portée. En effet, les professeurs en tant que médiateurs dans la construction du savoir scientifique, n'ont pas pu corriger les représentations des élèves avec beaucoup de succès ; cela est du au fait qu'ils ne tiennent pas compte de différentes représentations que se font les élèves à propos de certains concepts. On constate alors qu'une bonne partie d'élèves n'arrivent pas à réguler leurs représentations, ce qui explique pourquoi ne réussissent-ils pas à traduire les relations entre concepts à des lois ou à des formules mathématiques. 
En tenant compte de ce que nous avons signalé dans le cadre conceptuel à propos des perspectives de résolution de problèmes dans l'enseignement/apprentissage de la physique-chimie (Sall, 2002), nous pouvons dire que les deux perspectives "outil d'évaluation" et "activités d'apprentissage des concepts scientifiques" ne semblent pas être réalisées ici comme nous l'avons souhaité au départ. Ainsi, plusieurs apprenants n'arrivent pas d'une part à corriger leurs représentations après enseignement de manière à approfondir leurs esprits d'analyse et de raisonnement et d'autre part à apprendre les concepts scientifiques et les mettre en scène par l'application des certaines relations.

Malgré les efforts fournis par les professeurs, on constate la prédominance des activités monstratives qui consistent en un exposé du savoir, sous forme de rappels, en direction de la classe; celle-ci se contente généralement de suivre ce qui se fait au tableau. C'est donc l'élève au tableau et le professeur qui sont en activité; les autres élèves de la classe écoutent, regardent ce qui se fait au tableau, prennent notes parfois (ou recopient) dans leurs cahiers d'exercices. Cela entraîne des interactions à sens unique du professeur, ou de l'élève au tableau, vers le reste de la classe.

Les interactions élève-élève, pour autant qu'elles aient lieu (travail en groupe), sont assimilées à du bavardage même si de temps en temps cela a un rapport avec l'activité en question. Le professeur fait son boulot, mais est-ce que les élèves font leur travail, celui d'apprendre? Rien n'est moins sûr. Certes, il y a les efforts du professeur pour expliquer, répondre à des questions, procéder à des reformulations, interroger des élèves; mais il n'en demeure pas moins que le beau rôle lui revient. Pour s'en convaincre, il suffit que le professeur sorte pour voir les élèves se désintéresser complètement de l'activité et s'occuper d'autres choses qui les motivent davantage.

\subsection{Productions écrites (tâches)}

\subsubsection{Problème 2 (Tableau 2)}

La première question de ce problème correspond à l'item qui a obtenu le plus gros score ( 42 sur 50 , soit $84 \%$ ); on considère donc que cet item est réussi par la plus part des élèves. C'est le genre de questions qui permet aux élèves, même les plus faibles, d'entrer dans le problème. Les items 2 et 3 ont obtenu les mêmes scores 38 (76\%); ce qui peut être expliqué par le fait que l'élève pour qu'il puisse réaliser l'item 3 , doit utiliser l'item 2. Cela témoigne d'un esprit de raisonnement dont l'approfondissement est une finalité importante des activités de résolution de problème en physique-chimie (cadre conceptuel). On constate que le taux de réussite dépasse, pour les dix items proposés, les taux d'échec et de non traités, sauf pour l'item 9 "définir les stéréo-isoméries" dont on observe un taux de tâches non traitées de 24 sur 50, soit $48 \%$; ce taux est proche de celui de tâches réussies $(52 \%$, soit 26 sur 50 ) ; et pour l'item 4, c'est presque la même chose (réussies $52 \%$, échoués + non traités $=48 \%$ ).

En termes de compétence, nous pouvons dire que l'objectif est relativement réalisé puisque les élèves semblent être capables de mobiliser certaines ressources tout en choisissant, parmi les ressources qu'ils possèdent, celles qui conviennent à la situation qui leur est présentée. Néanmoins, la réussite 
relative dans ces items peut s'expliquer par le fait qu'ils font parti d'un algorithme que les élèves ont eu à répéter à plusieurs reprises ; ce qui témoigne que les élèves maîtrisent la méthodologie liée à ce type de problème. Par ailleurs, il semble que la diminution du taux de réussite de certaines tâches de ce problème dépend des difficultés des élèves au niveau de l'acquisition de mots clés (ex: non satureé) et de certaines questions (isomères de constitution). En tenant compte des échecs obtenus, nous pouvons dire que certains élèves aient des problèmes liés à la précision des questions; ce qui se traduit par le fait qu'ils sont arrivés à des réponses qui n’ont pas été demandées.

\subsubsection{Problème 3 (Tableau 3)}

$\mathrm{Si}$ on considère qu'un item est réussi par la classe quand le pourcentage d'élèves qui l'ont réalisé atteint $75 \%$ (soit 36 élèves), alors on décompte seulement un item réussi par la classe: l'item 2. Cependant les items réussis par au moins la moitié de la classe (soit 24 élèves ou plus) sont les items 3 et 4 en plus d'item 2. On remarque que les scores obtenus par les élèves pour les items 2 et 3 sont liés au fait que la relation déduite de la loi de maille comprend forcément les tensions à la borne des dipôles. De même les items 3 et 4 sont comparables, du point de vu score obtenu (24 et 26, respectivement), ce qui s'explique par le fait que l'item 4 pour qu'il soit réalisé par certaines élèves, ceux-ci devraient se servir de l'item 3. C'est pourquoi, un élève réussit les items 3 et 4 qui aident à une bonne représentation du problème et l'item 2 qui permet d'aboutir à la détermination des générateurs et les récepteurs sur le circuit; cet élève devrait aboutir à la solution malgré les obstacles que présentent certains items, notamment l'item 1 (score 12) et l'item 5 (score 8 soit $16 \%$ de réussite).

Ces obstacles sont peut être liés aux données qualitatives du problème et à certaines représentations qu'avaient les élèves sur certains concepts mis en jeu dans ce problème. Certains élèves se distinguent des autres par le calcul de la tension aux bornes du dipôle (BD); ils ont calculé $\mathrm{U}_{\mathbf{D B}}$ au lieu de calculer $\mathrm{U}_{\mathbf{B D}}$. En effet, le raisonnement utilisé par ces élèves n'est pas tellement faux, mais il résulte peut être des difficultés qu'ils avaient au niveau de la représentation de la tension; c'est pourquoi ces élèves ne parviennent pas à atteindre correctement l'objectif, qui consiste à déterminer les générateurs et les récepteurs sur le schéma en utilisant les conventions " récepteur" et "générateur".

Cependant, cette erreur pourrait être prévenue de la manière dont on formule les questions, les notations, et également le langage utilisé lors du processus enseignement apprentissage. On devrait donc préciser ce qu'on demande, faire attention au langage utilisé pour expliquer aux élèves ou pour leur poser des questions. En effet, il est plus judicieux de demander aux élèves de calculer précisément la tension $\mathrm{U}_{\mathrm{BD}}$ au lieu de leur demander de calculer la tension aux bornes de dipôle (BD); car cela pourrait aider aux mieux les élèves, ayant certaines ambigüités, de ne pas calculer $\mathrm{U}_{\mathrm{DB}}$ au lieu de $\mathrm{U}_{\mathrm{DB}}$.

Les items 7 et 8 sont réalisés avec des scores $22(45 \%)$ et $16(33 \%)$, respectivement. L'analyse des échecs des élèves concernant ces items nous permet de constater qu'ils font une confusion entre deux concepts 
complètement déférents qui sont la puissance consommée par le récepteur et celle délivrée par le générateur. Cela nous semble logique puisque l'item 5 concernant l'indication de la convention "récepteur" et "générateur" n'a été réalisé qu'avec un score très faible, soit $8(17 \%)$. D'où les difficultés auxquelles les élèves font face quand on leur demande de calculer la puissance consommée par le récepteur et celle délivrée par le générateur.

L'item 9 réalisé avec un score très faible, 10 soit $20 \%$, concerne une question où les élèves devaient élaborer et utiliser de schémas que de retenir des pré-requis. Généralement, si nous tenons compte de l'ensemble des items, le taux de réussite des élèves ici n'est que de $39 \%$, c'est un taux beaucoup plus faible de $75 \%$ que nous avons espéré et fixé comme un seuil de réussite de la classe pour chaque item.

Il se dégage de cette analyse que les élèves ne semblent pas accorder beaucoup d'importance, lors de la résolution d'un problème, à:

- L'énonciation des lois utilisées (score 12 soit $25 \%$ ): c'est une question qualitative qui renvoie à des difficultés d'ordre conceptuel, ou soit disant de la conception des élèves vis-à-vis des concepts physiques).

- L'application numérique (score 14) : c'est une question même si elle est quantitative, mais renvoie à des difficultés majeures qu'ont les élèves au niveau de l'utilisation des unités, notamment l'homogénéité de ces unités.

- L'indication des conventions (score 8).

Il est à noter que le problème 2 de chimie organique reste insuffisant pour tester les habilités des élèves lors de la résolution de problèmes en chimie car ce type de problèmes contient seulement des questions qualitatives, et non pas des questions quantitatives. Celles-ci, comme nous l'avons constaté en problème 3 de physique, présentent généralement des difficultés aux élèves non seulement au niveau du formalisme mathématique mais aussi au niveau des applications numériques et l'homogénéité des unités utilisées.

L'analyse des productions écrites des élèves et l'observation des séances de résolution de problèmes montrent que les élèves font face à plusieurs difficultés quand ils étaient en situation de résoudre un problème. Ainsi, nous avons pu formuler au moins trois types de difficultés:

- Difficultés résultant de la posture des élèves face aux données qualitatives qui sont soit négligées ou ignorées au profit des données quantitatives, soit mal interprétées, ce qui conduit à un faux chemin. D'ailleurs, les données qualitatives constituent la base sur laquelle s'appuie l'élaboration de la solution; les données quantitatives ne servent qu'à la fin du calcul littéral.

- Difficultés en lien avec les représentations des élèves qui faisaient obstacles à la compréhension des concepts utilisés dans les énoncés de problèmes (problèmes d'apprentissage des concepts dus à la non régulation des représentations). Quand on sait que l'énoncé est la porte d'entrée au problème, on comprend ce que ces représentations peuvent avoir comme conséquences si elles ne sont pas décelées et corrigées.

- Difficultés qui sont dues à l'incapacité, d'une très bonne partie d'élèves, à la traduction des phrases de contenu mathématique à des formules servant d'éléments essentiels à la résolution de problèmes en physique et chimie. 
Devant ces difficultés, il convient d'apporter quelques remèdes qui pourraient aider les élèves à les dépasser en:

- Faisant apprendre aux élèves la nécessité de lire et interpréter les consignes afin qu'ils puissent coder l'information de manière appropriée et systématique.

- Favorisant cher les élèves l'organisation de l'information: Savoir résoudre un problème renvoie à la capacité à sélectionner et à organiser l'information pertinente en une représentation globale et cohérente du problème.

- Stimulant chez les élèves la création et la vérification des stratégies: Une fois celles-ci apprises, elles deviennent une partie de la structure cognitive des élèves.

Le travail de groupes pourrait favoriser l'activité de résolution de problèmes et constituer ainsi une alternative pour améliorer les performances des élèves en résolution de problèmes. Les élèves en groupes s'interrogent entre eux sur les difficultés qu'ils rencontrent et s'entraident. De plus, ils peuvent échanger leurs idées, comparer leur méthode de résolution et vérifier leur résultat. Mais si le travail en groupes n'étaient pas bien organisé et animé, il serait contrairement une source de perturbations en classes.

\section{Conclusions}

La présente étude contribue à la compréhension des difficultés des élèves de première année Baccalauréat marocain dans les activités de résolution de problèmes en physique (le transfert d'énergie et le comportement global d'un circuit) ainsi qu'en chimie (la lecture d'une formule chimique).

Il ressort des analyses que les élèves ignorent ou négligent les données qualitatives des énoncés en faveur de celles quantitatives, avec comme conséquence un décalage au niveau du cheminement de résolution de problèmes. Les représentations initiales des élèves se dressent comme obstacles face aux concepts scientifiques à apprendre, tout comme l'incapacité de la plupart d'entre eux à traduire des contenus mathématiques en formules de résolution de problèmes. Aussi, les élèves semblent ne pas accorder beaucoup d'importance à l'énoncé des lois utilisées, à l'application numérique et à l'indication des conventions.

Il faut donc faire apprendre aux élèves la nécessité de lire et interpréter les consignes afin de pouvoir coder l'information pertinente et l'organiser en une représentation du problème, puis de stimuler chez eux la création et la vérification des stratégies.

\section{Bibliographic references}

CAILLOT, M. - DUMAS-CARRE, A. - GOFFARD, M. 1990. Le diagramme objets-interactions. Bulletin de l'union des physiciens, vol. 722, pp. 353-373. ISSN: 0366-3876.

CHI, M. T. H. - FELTOVITCH P. J. - GLASER R. 1981. Categorization and representation of physics problems by experts and novices. Cognitive Science. Vol.5 (2), pp.121-152. Online ISSN: 1551-6709

DE VECCHI, G. - CARMONA-MAGNALDI, N. 2002. Faire vivre de véritables situations problèmes. Paris : Hachette. ISBN 2.01.17.0852.4 
D'HAINAUT L. 1988. Des fins aux objectifs de l'éducation un cadre conceptuel et une méthode générale pour établir des résultats attendus d'une formation. (5eme éd.), Bruxelles: Editions Labor. ISBN : 2804003396 9782804003395

FABRE, M. 1999. Situation problèmes et savoirs scolaire. Paris: PUF. ISBN10: 2130496199 ISBN-13: 978-2130496199

FAGNANT, A. - VLASSIS J. 2010. Le rôle de la résolution de problèmes dans les apprentissages mathématiques: questions et réflexions. Education Canada, 50 (1), 50-52. ISSN: 0013-1253.

GAGNE, E. D. 1985. The cognitive psychology of school learning. Boston, Toronto: Little Brown Company. ISBN: 03163016559780316301657.

GIL PEREZ, D. 1996. New trends in science education. International Journal of Science Education, 18 (8), 889-901. Print ISSN: 0950-0693 Online ISSN: 1464-5289.

GIL PEREZ, D. - MARTINEZ-TERREGOZA, J. 1983. A model for problem-solving in accordance with scientific methodology. European Journal of Science Education, vol.5, pp.447-457. Print ISSN: 0950-0693 Online ISSN: 1464-5289.

GLOVER, J. A. - RONNING, R. R. - BRUNNING, R. H. 1990. Cognitive psychology for teachers. New York: Macmillian Publishing Company. ISBN10: 002344133X ISBN-13: 978-0023441332

GOFFARD, M. 1994. Le problème de physique et sa pédagogie. Paris: ADAPT-Snes. ISBN 2-909680-08-8.

LARKIN, J.-H. - RIEF, F. 1979. Understanding and learning problemsolving in physics. European Journal of Science Education vol.1, pp.191-203. Print ISSN: 0950-0693 Online ISSN: 1464-5289.

MAZOUZE, B. 2016. Des difficultés en résolution de problèmes de physique: quelles aides pour les élèves ? Educational Journal of the University of Patras UNESCO Chair, vol.3(2), pp. 258-268. ISSN: 2241-9152.

MAZOUZE, B. - LOUNIS, A. 2015. Résolution de problèmes et apprentissage des ondes : quels types de difficultés rencontrent les élèves ? Review of Science, Mathematics and ICT Education, vol. 9(2), pp. 25-40. ISSN: 1791-261X (print), 1792-3999 (electronic).

NEWELL, A. - SIMON, H. A. 1972. Human Problem Solving. Englewood Cliffs, New Jersey: Prentice-Hall. ISBN: 0134454030.

NOIRFALISE, N. - PORTE, J. 1990. Résolution de problème en second cycle. Repères, 1, 51-67. Print ISSN: 1157-1330 Online ISSN: 2263-5947.

PALLASCIO, R. 2005. Les situations-problèmes: un concept central du nouveau programme de mathématiques. Vie pédagogique, vol. 136, pp. 32-35. ISSN : 0707-2511.

POISSANT, H. - POELLHUBER, B. - FALAEDEAU, M. 1994. Résolution de problèmes, autorégulation et apprentissage. Revue canadienne de l'éducation vol. 19(1), pp. 30-44. Online ISSN: 1918-5979.

OUASRI, A. 2016. Study of the appropriation by pupils of second Baccalaureate year of knowledge objects relating to acide-bases titrations; Chemistry: Bulgarian Journal of Sciences Education, vol.25, n.6, pp. 695-717. ISSN (printed): 0861-9255. ISSN (electronic): 1313-8235. 
OUASRI, A. 2017a. Analyse des difficultés des élèves de deuxième année Baccalauréat marocain en résolution de problèmes de Mécanique; Educational Journal of the University of Patras UNESCO Chair, vol. 4(2), pp.39-57, ISSN : 2241-9152.

OUASRI, A. 2017b. Analyse des connaissances des de troisième année du collège marocain en activités de résolution de problèmes de l'électricité (loi d'Ohm, puissance et enérgie électrique); European Journal of Education Studies ; vol.3, Iss. 7, pp. 94-120. ISSN: 2501-1111.

OUASRI, A. 2017c. Study of Moroccan pupils' difficulties at second Baccalaureat year in solving chemistry problems relating to the reactivity of ethanoate ions and to Copper-Aluminium cell; Chemistry Education Research and Practice, vol. 18, pp. 737-748. Online only: ISSN 1756-1108.

REY, B. - CARETTE, V. - DEFRANCE, A. - KAHN, S. 2012. Les compétences à l'école. Apprentissage et évaluation. Bruxelles: De Boeck Education (3e édition) ISBN-10: 280417168X ISBN-13: 978-2804171681

ROBER, A. - MARC, W. 2013. Étude comparée de deux pratiques enseignantes lors d'une séquence de résolution d'un problème de modélisation. Éducation et didactique, vol. 7-3, pp. 55-78. ISSN : 1956-3485

RODITI, E. 2006. Les pratiques enseignantes en mathématiques: entre contraintes et liberté pédagogique. Paris: l'Harmattan. ISBN: 2-7475-7946-8.

SALL, C. T. 2002. L'auto-évaluation du profil d'entrée: une stratégie constructiviste dans la formation professionnelle initiale des enseignants. In Actes des 2èmes Assises du CIFFERSE: l'enseignement des sciences expérimentales. Dakar, 8-10 Avril 2001.

STREVELER, R. A. - LITZINGER, T. A. - MILLER, R. L. - STEIF, P. S. 2008. Learning conceptual knowledge in the engineering sciences: Overview and future research directions. Journal of Engineering Education, vol. 97, pp. 279-294. Online ISSN: 2168-9830

TARDIF, J. 1999. Pour un enseignement stratégique. L'apport de la psychologie cognitive. Montréal: Les éditions logiques. ISBN: 2-89381-0608

VAN DER SANDT, S. 2007. Research framework on mathematics teacher behaviour: Koehler and grows' Framework revisited. Eurasian Journal of Mathematics, Science \& Technology Education, vol. 3(4), pp. 343-350. EISSN: 1305-8223.

VERSCHAFFELL, L. - GREER, B. - DE CORTE, E. 2000. Making sense of word problem. Lisse, The Nethrlands: Swets and Zeitleinger. ISBN: 9789026516283.

WEIL-BARRAIS A. - LEMEIGNAN, G. 1990. Apprentissage de concepts en mécanique et modélisation de situations expérimentales. European Journal of Psychology, 5(4), 391-416. Print ISSN: 0256-2928 Online ISSN: 18785174. 


\section{Annexe}

\section{Annexe 1 :}

Tableau 1. Grille d'observation des séances de résolutions de problèmes animées par les professeurs.

\begin{tabular}{|c|c|c|}
\hline Niveaux d'observation & Oui & Non \\
\hline Le professeur a-t-il fait apparaître les objectifs de & & \\
\hline Le professeur a-t-il suscité l'intérêt des élèves pour & & \\
\hline Le professeur a-t-il encouragé la participation des & & \\
\hline Les élèves ont-ils participé de façon significative? & & \\
\hline Est-ce qu'un élève est envoyé au tableau et les autres le & & \\
\hline Est-ce que les élèves travaillent en groupes? & & \\
\hline Prennent-ils note au fur et à mesure ou attendent-ils la & & \\
\hline Posent-ils des questions au professeur? & & \\
\hline Posent-ils des questions à l'élève au tableau? & & \\
\hline Le professeur donne-t-il l'initiative aux élèves? & & \\
\hline Perçoit-on une progression dans la conduite de l'activité? & & \\
\hline Le professeur a-t-il stimulé l'apport des élèves? & & \\
\hline Les élèves ont-ils contribué en termes de propositions? & & \\
\hline Perçoit-on une méthode structurée dans la conduite de & & \\
\hline Le professeur tient-il compte de la représentation des & & \\
\hline L'activité se fait-elle avec des rappels de cours? & & \\
\hline Les élèves ont-ils contesté la réponse à une question? & & \\
\hline Le sens des concepts utilisés est-il rappelé ? & & \\
\hline Les relations entre concepts sont-elles données à travers & & \\
\hline Les données qualitatives sont-elles explicitées? & & \\
\hline
\end{tabular}




\section{Annexe 2}

\section{Problème 1 (Physique)}

On exprime l'intensité du champ magnétique B à l'intérieur d'une bobine, de longueur 1 et de rayon $r$, contenant $\mathrm{N}$ spires et traversé par un courant d'intensité I, par :

$$
B=\mu_{0} \frac{N I}{\sqrt{1^{2}+4 r^{2}}}
$$

1) Calculer $\mathrm{B}$ dans le cas suivant : $\mathrm{l}=2 \mathrm{r}=10 \mathrm{~cm} \mathrm{~N}=100$; $=1 \mathrm{~A}$

2) Quelle est l'erreur qu'on commit dans le cas où :

2.1. On considère la bobine plane

2.2. On considère la bobine comme un solénoïde

3) On considère $x=2 r / 1$

3.1. À partir de quelle valeur de $\mathrm{x}$ l'intensité du champ magnétique à l'intérieur de la bobine ne varie que de 1 si on considère la bobine plane

3.2. Même question si on considère la bobine comme un solénoïde.

\section{Problème 2 (Chimie)}

1) La formule générale d'un composé hydrocarbure non saturé est $\mathrm{C}_{4} \mathrm{H}_{8}$.

1.1. Déterminer le groupe organique auquel appartient ce composé en déterminant la différence entre les chaînes carboniques

1.2. Donner tous les isomères de formations possibles de ce composé

2) Le tableau suivant donne le pourcentage en masse d'un mélange de composés dont les chaînes carboniques contiennent cinq atomes de carbone résultants de la re-synthèse catalytique du pentane.

2.1. Donner les formules semi développés ainsi que l'écriture topologique des composés figurant dans le tableau.

2.2. Déterminer les isomères de forme (spatiale).

\begin{tabular}{|l|c|l|l|}
\hline \multicolumn{1}{|c|}{ Composés } & $\begin{array}{c}\text { Pourcentage } \\
\text { en masse }\end{array}$ & $\begin{array}{c}\text { Formule semi } \\
\text { développée }\end{array}$ & $\begin{array}{c}\text { Ecriture } \\
\text { topologique }\end{array}$ \\
\hline Pentane & 4 & & \\
\hline 2-methylbutane & 3 & & \\
\hline (Z) pent-2-ene & 8 & & \\
\hline (E) pent-2-ene & 12 & & \\
\hline $\begin{array}{l}\text { 2-methyl but-1- } \\
\text { ene }\end{array}$ & 11 & & \\
\hline $\begin{array}{l}\text { 3-methyl but-1- } \\
\text { ene }\end{array}$ & 5 & & \\
\hline $\begin{array}{l}\text { 2-methyl but-2- } \\
\text { ene }\end{array}$ & 13 & & \\
\hline Pent-1-ene & 33 & & \\
\hline Autres composés & 11 & & \\
\hline
\end{tabular}




\section{Problème 3 (Physique)}

1) Déterminer sur le circuit suivant les moteurs ainsi que les récepteurs de l'énergie électrique.

2) Calculer la puissance électrique consommée par les récepteurs, puis en déduire l'énergie libérée au circuit, de la part du moteur, par deux méthodes

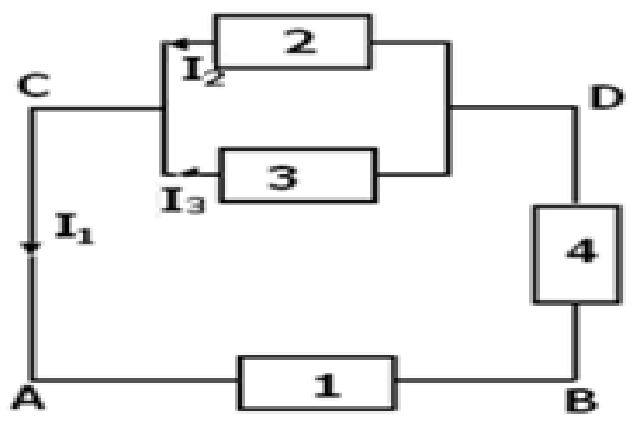

Prof. Dr. Ali Ouasri

Department of Physics and Chemistry

Regional Center of the Trades of Education and Training

Madinat Al Irfane, Souissi, BP 6210, Rabat,

Maroc

aouasri@yahoo.fr

Prof. Dr. Moulay Abderrahim EL MHAMMEDI

Chemistry Laboratory or Mathematics (Laboratoire de Chimie et Modélisation Mathématique - LCMM),

Polydisciplinary Faculty

University Hassan 1

BP 145, 25000 Khouribga

Maroc

elmhammedi@yahoo.fr 